See discussions, stats, and author profiles for this publication at: https://www.researchgate.net/publication/325225333

\title{
Busker Robot: a robotic painting system for rendering images into watercolour artworks
}

Conference Paper · May 2018

CITATION

1

4 authors, including:

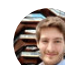

Lorenzo Scalera

University of Udine

38 PUBLICATIONS 52 CITATIONS

SEE PROFILE

Paolo Gallina

University of Trieste

102 PUBLICATIONS 1,335 CITATIONS

SEE PROFILE

Some of the authors of this publication are also working on these related projects:

Life Sciences View project

Space Robotics View project
READS

166

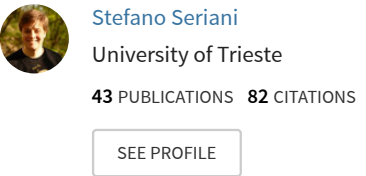




\title{
Busker Robot: a robotic painting system for rendering images into watercolour artworks
}

\author{
Lorenzo Scalera ${ }^{1}$, Stefano Seriani ${ }^{2}$, \\ Alessandro Gasparetto ${ }^{1}$, and Paolo Gallina ${ }^{2}$ \\ 1 University of Udine, Udine, Italy \\ scalera.lorenzo@spes.uniud.it, alessandro.gasparetto@uniud.it, \\ 2 University of Trieste, Trieste, Italy \\ sseriani@units.it, pgallina@uniuts.it
}

\begin{abstract}
In this paper we present Busker Robot, an innovative robotic painting system for rendering digital images into watercolour artworks. The installation is composed of a 6-degrees of freedom collaborative robot and a series of image processing and path planning algorithms. These non-photorealistic techniques elaborate a digital image into a sequence of trajectories that the robot reproduces on paper. Our painting machine is the first robotic system that uses watercolour technique for artistic rendering. The resulting artworks have been considered of interest by the public and the press in recent international fairs and exhibitions.
\end{abstract}

Keywords: robotic painting, watercolour, artistic rendering, image processing, path planning

\section{Introduction}

For thousands of years, from palaeolithic cave paintings to modern and contemporary arts, humans investigated several media to create representational figures and artistic expressions. In recent years, the robotic technology, usually employed in factories to enhance performance and production, has been adopted by artists and researchers to discover and experiment novel forms of art and painting.

Robotic painting is a complex and challenging process that comprises different fields, including mechanics of robots, control systems, image processing and art. Several examples of painting robots can be found in the present literature. One of the most important painting machine in contemporary art is AARON, designed by Harold Cohen in the '70s [1]. In most recent years, other examples can be given by the painting arm proposed by Aguilar et al. [2] and by the multifingered hands painting robot developed by Kudoh et al. [3]. One of the most impressive and fascinating artistic robots based on non-photorealistic rendering techniques is eDavid, developed by Deussen et al. [4]. Furthermore, Paul the Robot, created by Tresset and Fol Leymarie, is able to draw sketches of people using visual feedback to guide the drawing task [5]. Jain et al. developed a forcecontrolled robotic arm for portrait painting [6], Berio et al. proposed a compliant Baxter robot capable of drawing dynamic graffiti strokes [7], whereas Luo et al. 

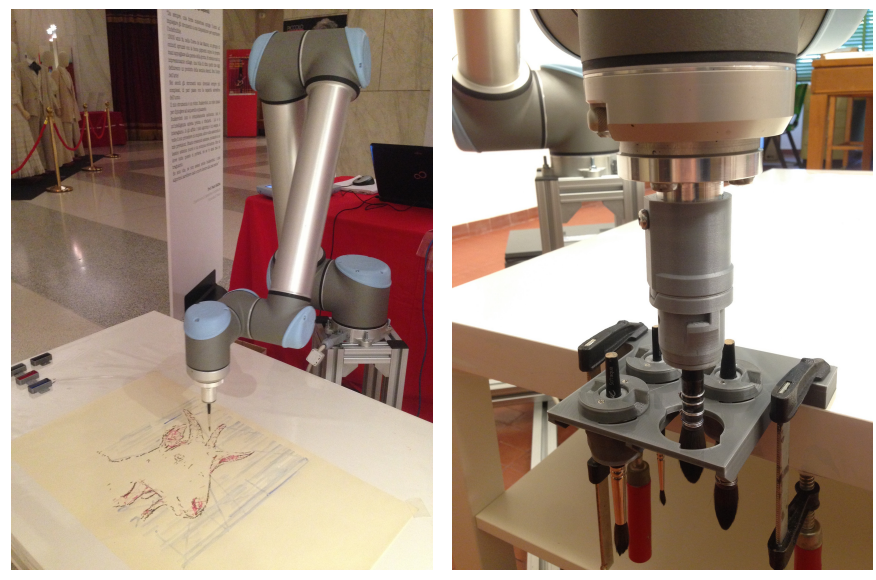

Fig. 1: (a) Busker Robot painting at "Piccolo Teatro" (Milan, Italy, November 2017), (b) automatic brush change system.

adopted a visual control system to guide their 7-DOFs robotic painting arm [8]. Applications of robot technology to art can be found in the automatic airbrush painting systems developed by Scalera et al. [9] and by Ago [10].

To the best of Authors knowledge, no examples of robotic painting systems using watercolour technique for artistic rendering can be found in literature. In this work, we propose a novel automatic system, called Busker Robot (Figure 1), that is capable of elaborating digital images and converting them into a series of trajectories that can be reproduced by a UR10 robot. The implemented algorithms are not aimed at faithfully reproduce the original image but to introduce a novel artistic contribution.

The paper is organized as follows: in Section 2 a brief overview on the watercolour technique is proposed, in Section 3 the architecture of the system is presented, whereas in Section 4 the experimental set-up is described. Section 5 reports on the implemented algorithms for artistic rendering. Finally, in Section 6 the experimental results are analysed and in Section 7 the conclusion and the future developments of this work are reported.

\section{Watercolour Technique}

Watercolour painting is an ancient technique in which fine pigments are suspended in a water-based solution. Its origins date perhaps to the palaeolithic paintings but, during the centuries, it is has been adopted in Egyptian and Oriental Art. In modern history, watercolour is related to artists such as T. Girtin and J.M.W. Turner, that adopted this technique in their expressive and turbulent landscapes. In the present literature, several contributions aimed at characterizing and simulating watercolour effects can be found. Interesting examples are given by the work of Curtis el at. [11], who presented a model for interesting 
watercolour rendering, and by Montesdeoca et al. [12], who investigated different effects of watercolour painting for animations and graphics.

In contrast with opaque paints used in oil and acrylic techniques, the properties of watercolour are related to the semi-transparent suspension of pigments in water and to the interaction between watered paint and paper. Watercolour allows to apply on the paper several layers of colour each one over others, it allows the colours to be mixed and diluted, generating uncontrollable effects such as backruns, granulation, flow patterns and edge-darkening [11]. The brushing technique influences the outcome as well with effects due to the application of watered paint on dry or wet paper, or of a nearly-dry brush on rough paper.

It can be noticed how complex and challenging the automation of watercolour painting could be. In fact, not only the colour dilution but also the choice of paper, brushes and stroke parameters is extremely important for the painting task. For these reasons, in a preliminary stage, a characterization of strokes and brushes is needed in order to fully understand and control the process.

\section{Busker Robot}

In this Section a brief overview on the robotic painting system is presented. Busker Robot is composed of both hardware (the painting machine) and software elements (the algorithms for artistic rendering and trajectory planning). The algorithms, that will be explained in Section 5, elaborate an input image by applying several non-photorealistic techniques aimed at extracting and processing the large areas and backgrounds as well the details and contours. The extracted features are then converted into a series of paths for the robot. Then, the resulting trajectories are executed by the robotic arm and translated into the artwork. In Figure 2 a graphical overview on the system is reported.

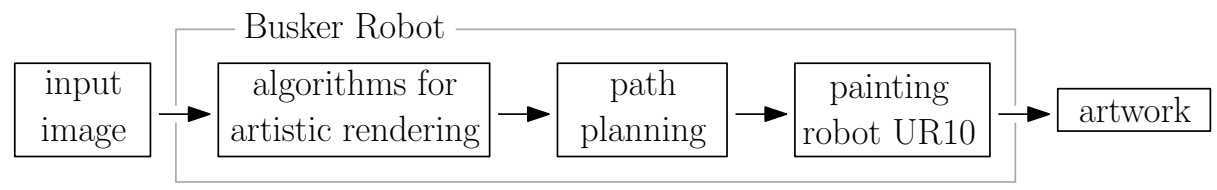

Fig. 2: Graphical overview on the robotic painting system.

\section{Experimental Set-Up}

The painting machine used in this work is a 6-DOFs UR10 collaborative robot, by Industrial Robots, that we equipped for painting purposes. An image of the robot performing watercolour painting is reported in Figure 1(a). The robot is intrinsically safe since it is provided with collision-detecting systems and force limits and, therefore, the artist controlling the system can operate side by side 
with it changing the colours, adjusting the dilution and monitoring the operations. Prior to starting the painting the robot has to be aware of the surrounding environment, in particular of the position of paint cups, paper and brush tool repository. Furthermore, a calibration of the drawing surface is applied in order to avoid planarity errors between the robot base and the paper support.

Watercolour painting is performed using Raphaël Soft Aqua brushes, characterized by a high fluid retention capacity and, therefore, ideal for the application. The brushes have been equipped with 3D printed supports, that allow to be easily integrated with an aluminium flange mounted on the robot end-effector. Moreover, an automatic brush change system has been developed (Figure 1(b)) in order to paint strokes with variable thickness, using different brushes. The brush change system is composed by a fixed support and a removable coupler, one for each of the four brushes. Fixed and removable couplers have been printed in PLA with an Ultimaker 2+ and they are joined by means of cylindrical magnets, that allow an easy pick up and release of the brush tool from the repository with a simple rotation of the robot sixth joint.

The brushing mechanics has been analysed in order to determine the variability of two main parameters: the stroke width and the colour intensity along the stroke. Indeed, because of the inherent complexity of the interaction between the brush and the paper, the deposition of paint on the surface is hard to model. Even though the characterization of the strokes is out of the scope of this paper, in order to provide some indication on its morphology, we can generally affirm that the evolution of the intensity level expresses a decrease along the strokes as well as the thickness. Furthermore, both parameters show a small increase during the final part of a stroke. This is likely due to the speed profile of the robot trajectory, which is effectively trapezoidal, having a deceleration phase at the end; as it is expected, this indicates that slower brushing speeds cause increased deposition of paint per linear distance.

\section{Non-Photorealistic Rendering Techniques}

In this Section, we describe the Non-Photorealistic Rendering Techniques implemented in this work, also referred as Image-Based Artistic Rendering [13] as the initial input is a 2D image. The algorithms for artistic rendering presented in the following are Random Strokes, used for the covering of backgrounds and large areas, and the Difference of Gaussians, suitable for contours and small detailed parts. As explained in Figure 2, the input of these algorithms is a digital image and the output is the set of points that describes the extracted image features. Then, the path planning module elaborates these points into a series of trajectories for the robot, including the motions for the paint refill, the brush change and draining and, finally, deploys the script file on the UR10. The algorithms

have been implemented as graphical user interfaces in Matlab by adopting the UR Script Programming Language to control the robot. 


\subsection{Random Strokes}

The Random Strokes technique has been implemented for the elaboration of backgrounds and large areas of an image. The technique is based on a greyscale threshold operation: given a specific intensity level $I_{T}$, each pixel $P_{i}$ of the original image, with intensity $I\left(P_{i}\right)$, is converted in a binary number equal to 1 when $I\left(P_{i}\right) \geq I_{T}$ and to 0 otherwise. Then, a user-defined number $N$ of points is randomly generated inside the area. For each point $Q_{i}(x, y)$ the orientation $\theta(x, y)$ of the grey-scale gradient $G=\left(G_{x}, G_{y}\right)$ is calculated as:

$$
\theta(x, y)=\arctan \left(G_{y}, G_{x}\right)
$$

Starting from each point $Q_{i}(x, y)$, the algorithm generates a straight line perpendicular to $\theta(x, y)$ that continues through the area till the border is found. The algorithm can be applied by choosing several different thresholds, each of them consisting in gradient-based random lines overlapping one to each other. The random generation of initial points ensures that a different result is obtained each time the algorithm is run; in this manner each configuration is unique and particular. In Figures 3(b) and 4(b) two example obtained by the Random Stroke are shown. In the rendering, the line thickness does not represent the real strokes thickness on the paper: it is correlated with the brush size, the vertical position of the brush with respect to the paper and with the scaling factor.

\subsection{Difference of Gaussians}

The Difference of Gaussians (DOG) $[14,15]$ is an image processing algorithm for edge detection. It is based on the Gaussian blur of a grey-scale image at a specified standard deviation $\sigma$ and window size, that suppresses only high-frequency spatial information. The blurred version of the original image is then subtracted from another, less blurred version of the original (obtained with a sharper $\sigma$ ). The resulting image is then calculated by replacing each pixel with the value of the DOG function and detecting the zero-crossing points (i.e. negative becomes positive and vice versa). A threshold is then applied to the zero-crossings in order to keep only the stronger ones, which represent the final detected edges of the image. In the case of standard deviations $\sigma_{1}$ and $\sigma_{2}\left(\sigma_{2}>\sigma_{1}\right)$, the Difference of Gaussians function $\Gamma_{\sigma_{1}, \sigma_{2}}(x, y)$ of the image $I(x, y)$, can be calculated as:

$$
\Gamma_{\sigma_{1}, \sigma_{2}}(x, y)=I(x, y) \frac{1}{2 \pi \sigma_{1}^{2}} e^{-\frac{x^{2}+y^{2}}{2 \sigma_{1}^{2}}}-I(x, y) \frac{1}{2 \pi \sigma_{2}^{2}} e^{-\frac{x^{2}+y^{2}}{2 \sigma_{2}^{2}}}
$$

Examples of images elaborated with the Difference of Gaussians algorithm can be found in Figures 3(c) and 4(c), obtained by elaborating the images of the goat and Paolini respectively. It has to be noticed that for each starting image, different results can be found by modifying the Gaussian function parameters as well as the threshold. Furthermore, a filter could be applied to the final strokes, e.g. to eliminate the shortest ones, before applying the path planning algorithm. 


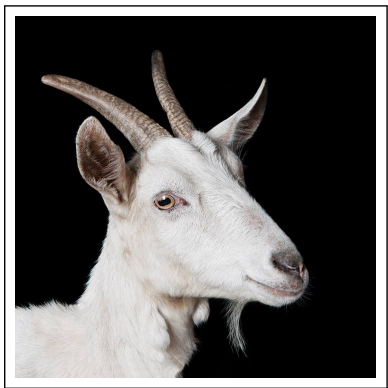

(a)

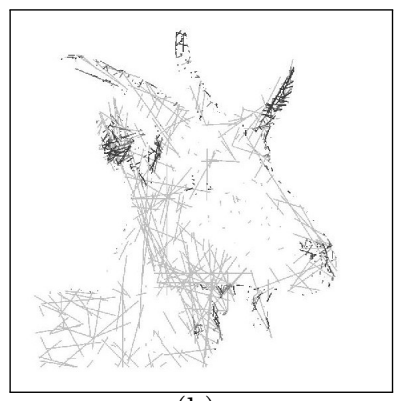

(b)

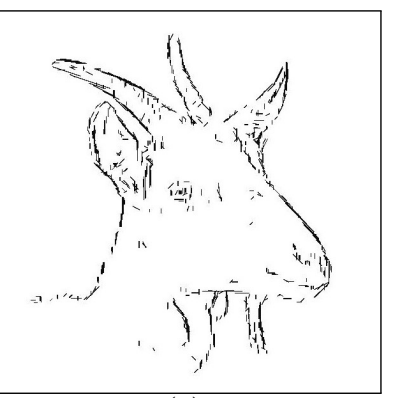

(c)

Fig. 3: Goat: (a) original image, (b) Random Strokes, (c) Difference of Gaussians.

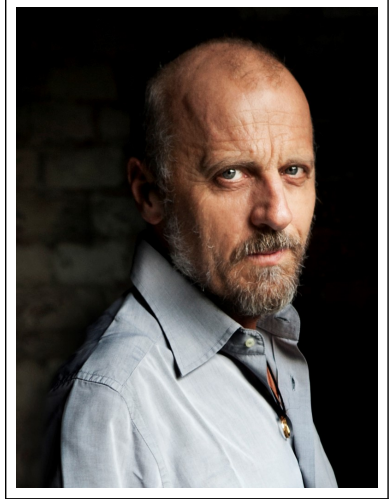

(a)

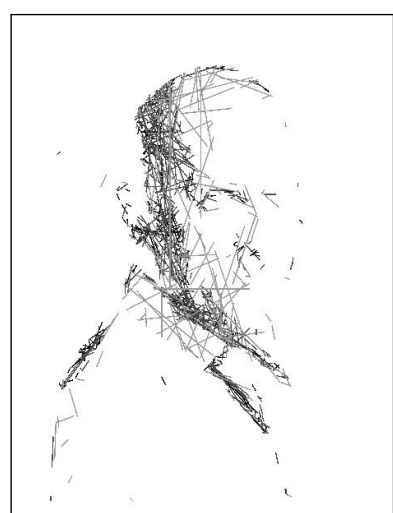

(b)

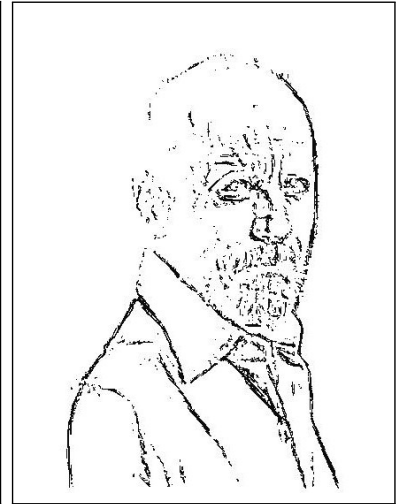

(c)

Fig. 4: Paolini: (a) original image (photo courtesy of Marco Paolini), (b) Random Strokes, (c) Difference of Gaussians.

\section{Experimental Results}

Two examples of resulting artworks, obtained with the combination of the techniques described in the previous sections, are reported in Figure 5. In particular, for both the goat (Fig.5(a)) and Paolini (Fig.5(b)), the Random Strokes algorithm has been applied for the background and for the dark areas, whereas the Difference of Gaussians for the main details and contours. It has to be noticed that, in the context of robotic painting and even more in the challenging watercolour technique, a quantitative analysis of the results is not possible. This is because the appreciation of an artwork is personal and subjective. Nevertheless, the artworks produced by Busker Robot are unique in their watercolour expression and they raised interest and fascination in recent international fairs and events. 


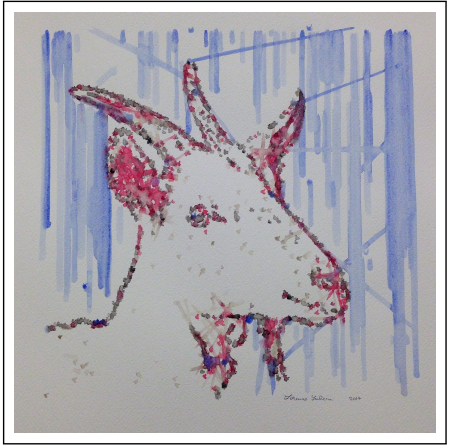

(a)

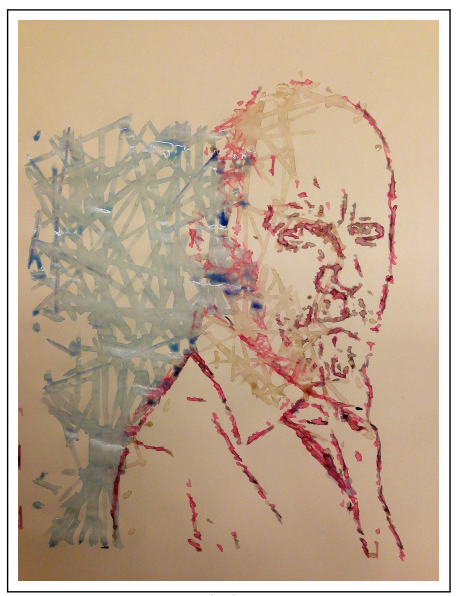

(b)

Fig. 5: Resulting artworks: (a) goat and (b) Paolini.

\section{Conclusion}

In this paper we presented Busker Robot, an innovative robotic painting system for rendering digital images into watercolour artworks. The system is composed of a 6-DOFs UR10 robot, equipped for painting purposes, and a series of artistic rendering and path planning algorithms. Several non-photorealistic techniques have been implemented to extract the large areas and the details of the input image and elaborate them into a series of paths for the robot. In particular, the Random Strokes and the Difference of Gaussians algorithms have been described in this paper. Busker Robot is the first robotic painting system that uses watercolour technique for artistic rendering. The resulting artworks have been appreciated and considered of interest by the public and the local and international press in recent international fairs and exhibitions [16]. In future, we plan to further investigate watercolour robotic painting, by implementing new algorithms, improving the brushing technique and adopting a visual feedback system to better control the painting in progress.

\section{Acknowledgements}

The work presented in this paper has been supported by Fondo Ricerca Ateneo, FRA 2015 (Internal Fund, University of Trieste).

\section{References}

1. H. Cohen, "The further exploits of AARON, painter," Stanford Humanities Review, vol. 4, no. 2, pp. 141-158, 1995. 
2. C. Aguilar and H. Lipson, "A robotic system for interpreting images into painted artwork," in International Conference on Generative Art, vol. 11, 2008.

3. S. Kudoh, K. Ogawara, M. Ruchanurucks, and K. Ikeuchi, "Painting robot with multi-fingered hands and stereo vision," Robotics and Autonomous Systems, vol. 57, no. 3, pp. 279-288, 2009.

4. O. Deussen, T. Lindemeier, S. Pirk, and M. Tautzenberger, "Feedback-guided stroke placement for a painting machine," in Proceedings of the 8th Annual Symposium on Computational Aesthetics in Graphics, Visualization, and Imaging. Eurographics Association, 2012, pp. 25-33.

5. P. Tresset and F. F. Leymarie, "Portrait drawing by Paul the robot," Computers Es Graphics, vol. 37, no. 5, pp. 348-363, 2013.

6. S. Jain, P. Gupta, V. Kumar, and K. Sharma, "A force-controlled portrait drawing robot," in Industrial Technology (ICIT), 2015 IEEE International Conference on. IEEE, 2015, pp. 3160-3165.

7. D. Berio, S. Calinon, and F. F. Leymarie, "Learning dynamic graffiti strokes with a compliant robot," in Intelligent Robots and Systems (IROS), 2016 IEEE/RSJ International Conference on. IEEE, 2016, pp. 3981-3986.

8. R. C. Luo, M.-J. Hong, and P.-C. Chung, "Robot artist for colorful picture painting with visual control system," in Intelligent Robots and Systems (IROS), 2016 IEEE/RSJ International Conference on. IEEE, 2016, pp. 2998-3003.

9. L. Scalera, E. Mazzon, P. Gallina, and A. Gasparetto, "Airbrush robotic painting system: Experimental validation of a colour spray model," in International Conference on Robotics in Alpe-Adria Danube Region. Springer, 2017, pp. 549-556.

10. V. Ago, "Robotic airbrush painting," http://violaago.com/ robotic-airbrush-painting/, 2013.

11. C. J. Curtis, S. E. Anderson, J. E. Seims, K. W. Fleischer, and D. H. Salesin, "Computer-generated watercolor," in Proceedings of the 24th Annual Conference on Computer Graphics and Interactive Techniques. ACM Press/Addison-Wesley Publishing Co., 1997, pp. 421-430.

12. S. Montesdeoca, H. S. Seah, P. Bénard, R. Vergne, J. Thollot, H.-M. Rall, and D. Benvenuti, "Edge-and substrate-based effects for watercolor stylization," in Expressive 2017, Symposium on Computational Aesthetics, Sketch-Based Interfaces and Modeling, and Non-Photorealistic Animation and Rendering, 2017, p. 10.

13. J. E. Kyprianidis, J. Collomosse, T. Wang, and T. Isenberg, "State of the" art: A taxonomy of artistic stylization techniques for images and video," IEEE Transactions on Visualization and Computer Graphics, vol. 19, no. 5, pp. 866-885, 2013.

14. D. Marr and E. Hildreth, "Theory of edge detection," in Proc. R. Soc. Lond. B, vol. 207, no. 1167. The Royal Society, 1980, pp. 187-217.

15. S. Wang, W. Li, Y. Wang, Y. Jiang, S. Jiang, and R. Zhao, "An improved Difference of Gaussian filter in face recognition." Journal of Multimedia, vol. 7, no. 6, pp. 429433, 2012.

16. "aNEWS, Italian prof. creates robotic artist," http://www.anews.com.tr/webtv/ life/italian-prof-creates-robotic-artist/, 2017. 